Comment. Math. Helv. 72 (1997) 72-83

0010-2571/97/010072-12\$1.50+0.20/0
(C) 1997 Birkhäuser Verlag, Basel

Commentarii Mathematici Helvetici

\title{
On the Betti number of the image of a generic map
}

\author{
Carlos Biasi and Osamu Saeki*
}

\begin{abstract}
Let $f: M \rightarrow N$ be a differentiable map of a closed $m$-dimensional manifold into an $(m+k)$-dimensional manifold with $k>0$. We show, assuming that $f$ is generic in a certain sense, that $f$ is an embedding if and only if the $(m-k+1)$-th Betti numbers with respect to the Cech homology of $M$ and $f(M)$ coincide, under a certain condition on the stable normal bundle of $f$. This generalizes the authors' previous result for immersions with normal crossings [BS1]. As a corollary, we obtain the converse of the Jordan-Brouwer theorem for codimension-1 generic maps, which is a generalization of the results of [BR, BMS1, BMS2, Sae1] for immersions with normal crossings.
\end{abstract}

Mathematics Subject Classification (1991). Primary 57R35; Secondary 57R40, 57R45, $55 \mathrm{~N} 05$.

Keywords. Betti number, generic map, Čech homology, Jordan-Brouwer theorem, ANR.

\section{Introduction}

Let $f: M \rightarrow N$ be a differentiable map of a closed $m$-dimensional manifold into an $n$-dimensional manifold with $k=n-m>0$. In [BS1], assuming that $f$ is an immersion with normal crossings, the authors have shown that $f$ is a differentiable embedding if and only if the $(m-k+1)$-th Betti numbers with respect to the singular homology of $M$ and $f(M)$ coincide and a certain pair of cohomology classes in $H^{k}\left(M ; \mathbf{Z}_{2}\right)$ determined by $f$ coincide. In the course of the proof, we have essentially used a formula originally due to Whitney [Wh] (see also [He]) which describes the homology class represented by the self-intersection set of $f$.

The purpose of this paper is to generalize the above mentioned result to generic differentiable maps in the sense of Ronga $[R]$. For a precise statement, see $\S 2$ (Theorem 2.2). In fact, Ronga has given a formula for the homology class represented by the closure of the self-intersection set of a generic map, and this formula has enabled us to generalize the previous result. However, a straightforward gener-

\footnotetext{
*The second author is partly supported by CNPq, Brazil, and by Grant-in-Aid for Encouragement of Young Scientists (No. 08740057), Ministry of Education, Science and Culture, Japan.
} 
alization has not been easy, mainly because of bad topological behaviors of the image $f(M)$. When $f$ is an immersion with normal crossings, the image $f(M)$ has a natural stratification into multiple point sets and, in particular, it is triangulable. However, generally speaking, the image $f(M)$ is not even an ANR (absolute neighborhood retract), even if $f$ is generic in the sense of Ronga. Thus, instead of the usual Betti numbers with respect to the singular homology, we have used the Betti numbers with respect to the Čech homology.

As a corollary to our characterization of embeddings, we obtain a converse of the Jordan-Brouwer theorem for codimension-1 generic maps. In other words, under a certain homological condition, we show that a generic differentiable map $f: M \rightarrow N$ with $\operatorname{dim} N=\operatorname{dim} M+1$ is an embedding if and only if the image $f(M)$ of $f$ separates $N$ into exactly two connected components (see Corollary 2.6 and Theorem 3.6). Since immersions with normal crossings are generic, this generalizes the previous results in [BR, BMS1, BMS2, Sae1].

The paper is organized as follows. In $\S 2$, we state the main theorems and the corollaries in a precise manner. We give the proofs of these theorems and corollaries in $\S 3$. We also mention a result (Remark 3.7) about the $k$-th Betti number of the complement of the image of a generic map, which is related to a result of Hirsch [Hi]. In $\S 4$, in order to convince the reader that a generic map can behave badly, we give an example of generic maps whose images are not ANR's.

Throughout the paper, the homology and cohomology groups have $\mathbf{Z}_{2}$ coefficients unless otherwise indicated. All manifolds are of class $C^{\infty}$, paracompact and Hausdorff.

This work has been done during the second author's stay in ICMSC-USP, Instituto de Ciências Matemáticas de São Carlos, Universidade de São Paulo, Brazil. He would like to thank the people there for their hospitality and for many stimulating discussions. The authors would like to express their thanks to the referee for his suggestions, especially for his alternative proof of our Lemma 3.1.

\section{Statement of the main results}

Let $f: M \rightarrow N$ be a continuous map of an $m$-dimensional manifold $M$ into an $n$-dimensional manifold $N$. We suppose that $k=n-m>0$ and that the map $f$ is proper. For the moment, we assume no differentiability of $f$. Let the stable normal bundle $f^{*} T N \oplus \nu_{M}$ of $f$ be denoted by $\nu_{f}$, where $\nu_{M}$ is the stable normal bundle of the manifold $M$. Then we denote by $w_{k}(f)\left(\in H^{k}(M)\right)$ the $k$-th Stiefel-Whitney class of the stable vector bundle $\nu_{f}$. Furthermore, we define $v(f) \in H^{k}(M)$ to be the image of the fundamental class $[M] \in H_{m}^{c}(M)$ by the composite

$$
H_{m}^{c}(M) \stackrel{f_{*}}{\longrightarrow} H_{m}^{c}(N) \stackrel{D_{N}^{-1}}{\longrightarrow} H^{k}(N) \stackrel{f^{*}}{\longrightarrow} H^{k}(M),
$$

where $H_{*}^{c}$ denotes the (singular) homology of the compatible family with respect 
to the compact subsets ([Sp, Chapter 6 , Section 3]), and $D_{N}$ denotes the Poincaré duality isomorphism.

We note that when $f$ is a differentiable immersion, the above definitions of $w_{k}(f)$ and $v(f)$ coincide with those of $w_{k}\left(\nu_{f}\right)$ and $v_{k}(f)$ respectively given in [BS1]. See also [LS] and [He, Proposition 4.1]. We also note that, if $f$ is a differentiable embedding, then $w_{k}(f)=v(f)$, as has been seen in [BS1] (see also [He] and [MS, Corollary 11.4]). As to the homotopy (or bordism) invariance of $w_{k}(f)$ and $v(f)$, see [BS2, BS3].

Next we define the class of differentiable maps which we are going to treat in this paper.

Definition 2.1. Let $f: M \rightarrow N$ be a proper map of class $C^{2}$ with $\operatorname{dim} M<$ $\operatorname{dim} N$. We say that $f$ is generic for the double points, if it is so in the sense of Ronga [R, Définition (p. 228)]; in other words, if the 1-jet extension $j^{1} f: M \rightarrow$ $J^{1}(M, N)$ of $f$ is transverse to the submanifolds $\Sigma^{i}=\left\{\alpha \in J^{1}(M, N) \mid \operatorname{dim} \operatorname{ker} \alpha=\right.$ $i$ \} for all $i$ and if the $l$-fold product map $f^{l}: M^{l} \rightarrow N^{l}$ is transverse to the diagonal $\delta_{N}^{l}$ of $N^{l}$ off the super diagonal $\Delta_{M}^{l}=\left\{\left(x_{1}, \ldots, x_{l}\right) \in M^{l} \mid x_{i}=x_{j}\right.$ for some $\left.i \neq j\right\}$ of $M^{l}$ for all $l=2,3,4, \ldots$.

Note that the set of the proper maps of class $C^{r}(2 \leq r \leq \infty)$ which are generic for the double points is dense in the space $C_{\mathrm{pr}}^{r}(M, N)$ of all proper maps of class $C^{r}$ of $M$ into $N$ with the Whitney $C^{r}$-topology.

In the following, $\check{H}^{*}$ and $\check{H}_{*}$ will denote the Čech (or Alexander-Čech) cohomology and homology respectively (see [ES, Sp, Wa, Gr], for example). For a topological space $X, \breve{\beta}_{i}(X)$ will denote the dimension of the vector space $\check{H}_{i}(X)$ over $\mathbf{Z}_{2}$. Here we note that $\check{H}_{*}$ and $\check{H}^{*}$ are naturally isomorphic to the singular homology and cohomology respectively for an ANR. In particular, this is valid for manifolds. We denote by $\beta_{i}(X)$ the dimension of the singular homology $H_{i}(X)$ and by $\tilde{\beta}_{i}(X)$ the dimension of the reduced singular homology $\tilde{H}_{i}(X)$.

The main result of this paper is the following.

Theorem 2.2. Let $f: M \rightarrow N$ be a map of class $C^{2}$ which is generic for the double points, where $M$ is a closed $m$-dimensional manifold and $N$ is an n-dimensional manifold with $k=n-m>0$. Then $f$ is a differentiable embedding if and only if $w_{k}(f)=v(f)$ and $\check{\beta}_{m-k+1}(M)=\check{\beta}_{m-k+1}(f(M))$.

The following is a direct consequence of Theorem 2.2 and the definition of $v(f)$.

Corollary 2.3. Let $f: M \rightarrow N$ be a map of class $C^{2}$ which is generic for the double points, where $M$ is a closed m-dimensional manifold and $N$ is an $n$ dimensional manifold with $k=n-m>0$. Suppose that either $f^{*}: H^{k}(N) \rightarrow$ $H^{k}(M)$ or $f_{*}: H_{m}(M) \rightarrow H_{m}(N)$ is the zero map. Then $f$ is a differentiable embedding if and only if $w_{k}(f)=0$ and $\check{\beta}_{m-k+1}(M)=\check{\beta}_{m-k+1}(f(M))$. 
As to the Betti number of the complement of the image of a map which is generic for the double points, we have the following.

Corollary 2.4. Let $f: M \rightarrow N$ be a map of class $C^{2}$ which is generic for the double points, where $M$ is a closed m-dimensional manifold and $N$ is an $n$ dimensional manifold with $k=n-m>0$. Suppose that $\beta_{k}(N)=\beta_{2 k-1}(N)=$ $\tilde{\beta}_{2 k-2}(N)=0$. Then $f$ is a differentiable embedding if and only if $w_{k}(f)=0$ and $\tilde{\beta}_{2 k-2}(N-f(M))=\beta_{k-1}(M)$.

Remark 2.5. When $N=\mathbf{R}^{n}$, we have the following: a map $f: M \rightarrow \mathbf{R}^{n}$ of class $C^{2}$ which is generic for the double points of a closed $m$-dimensional manifold $M$ with vanishing $k$-th dual Stiefel-Whitney class $\bar{w}_{k}(M)\left(\in H^{k}(M)\right)$ is an embedding if and only if $\tilde{\beta}_{2 k-2}\left(\mathbf{R}^{n}-f(M)\right)=\beta_{k-1}(M)$.

In the codimension- 1 case (i.e., the case with $k=1$ ), we have the following converse of the Jordan-Brouwer theorem for maps which are generic for the double points, which generalizes the results of [BR, BMS1, BMS2, Sae1].

Corollary 2.6. Let $f: M \rightarrow N$ be a map of class $C^{2}$ which is generic for the double points, where $M$ is a closed orientable $m$-dimensional manifold and $N$ is a connected $(m+1)$-dimensional manifold with $H_{1}(N)=0$. Then $f$ is a differentiable embedding if and only if $\beta_{0}(N-f(M))=\beta_{0}(M)+1$.

Note that, in the above corollary, $w_{1}(f)$ always vanishes, since $M$ and $N$ are orientable. Compare Corollary 2.6 with [Sae2]. See also Theorem 3.6 of the present paper.

\section{Proof of the main theorem}

Proof of Theorem 2.2. Let $f: M \rightarrow N$ be a map of class $C^{2}$ which is generic for the double points. Set $M(f)=\left\{x \in M \mid f^{-1}(f(x)) \neq\{x\}\right\}$ and $\Sigma(f)=\{x \in$ $\left.M \mid \operatorname{dim} \operatorname{ker} d f_{x} \geq 1\right\}$, which are called the self-intersection set and the singular set of $f$ respectively. Then, by Ronga [R, Théorème 2.5], the closure $A=\overline{M(f)}$ of $M(f)$ coincides with $M(f) \cup \Sigma(f)$. (Here we note that, in [R], maps are assumed to be of class $C^{\infty}$. However, the same argument works also for maps of class $C^{2}$.)

First we suppose that $f$ is not a differentiable embedding. Then $A$ is not empty. Consider the following commutative diagram: 


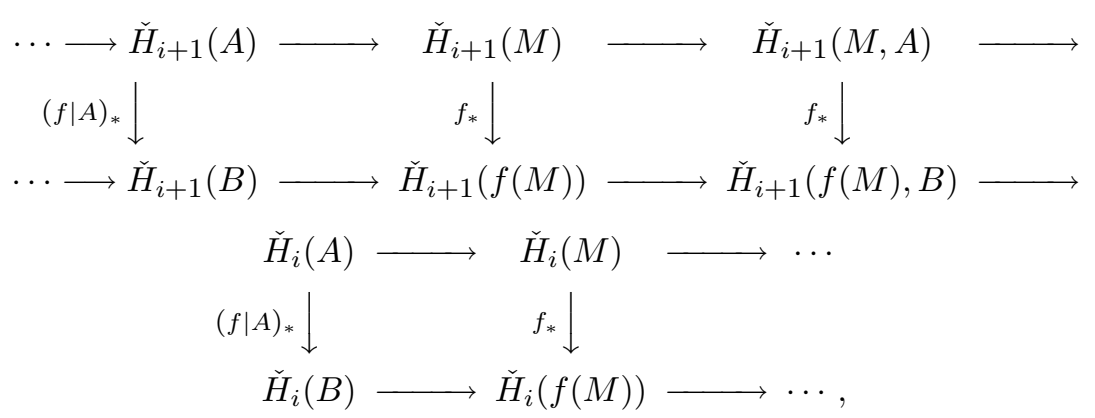

where $B=f(A)$. Note that each row is exact, since $(M, A)$ and $(f(M), B)$ are compact pairs (see [K, ES]). Furthermore, since $f \mid M-A: M-A \rightarrow f(M)-B$ is a homeomorphism, we see that $f_{*}: \check{H}_{i}(M, A) \rightarrow \check{H}_{i}(f(M), B)$ is an isomorphism for each $i$ (see [ES, Chapter X, $\S 5]$ ). Hence, by a standard argument, we have the following exact sequence:

$$
\begin{aligned}
\check{H}_{m-k+1}(A) & \rightarrow \check{H}_{m-k+1}(B) \oplus \check{H}_{m-k+1}(M) \rightarrow \check{H}_{m-k+1}(f(M)) \\
& \rightarrow \check{H}_{m-k}(A) \stackrel{\alpha}{\longrightarrow} \check{H}_{m-k}(B) \oplus \check{H}_{m-k}(M),
\end{aligned}
$$

where $\alpha=(f \mid A)_{*} \oplus j_{*}$ and $j: A \rightarrow M$ is the inclusion map (for example, see [D, p. 2]). Since $A$ is the image of a closed $(m-k)$-dimensional manifold by a differentiable map $([\mathrm{R}]$ ), we see that the topological dimension (for a definition, see [HW]) of $A$ is at most $m-k$ (see [Sar, Theorem 2 (p. 173)] or [C, Proposition 4]). Hence we have $\check{H}_{m-k+1}(A)=0$ (see [HW, Theorem VIII 4 (p. 152)]). We also have $\check{H}_{m-k+1}(B)=0$, since $B$ is the image of a closed $(m-k)$-dimensional manifold by a composite of two differentiable maps. Thus we have the exact sequence

$$
\begin{gathered}
0 \rightarrow \check{H}_{m-k+1}(M) \rightarrow \check{H}_{m-k+1}(f(M)) \\
\rightarrow \check{H}_{m-k}(A) \stackrel{\alpha}{\longrightarrow} \check{H}_{m-k}(B) \oplus \check{H}_{m-k}(M) .
\end{gathered}
$$

By $[\mathrm{R}]$, there exists a non-zero fundamental class $[A] \in H_{m-k}(A)$ in the singular homology such that there exists an open dense subset $U$ of $A$ which is a manifold of dimension $m-k$ and that the image of $[A]$ in $H_{m-k}(A, A-x) \cong \mathbf{Z}_{2}$ is the generator for all $x \in U$. Now consider the following commutative diagram:

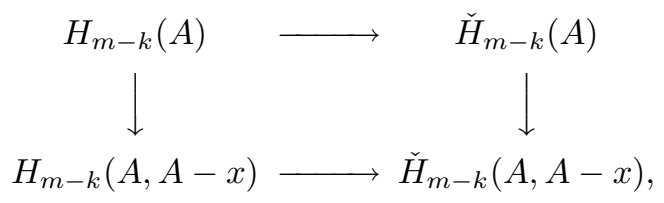

where the horizontal homomorphisms are the natural ones and the vertical ones are induced by the inclusions. Then we see that the lower horizontal homomorphism is an isomorphism by excision and hence that $[A]$ is non-zero also in $\breve{H}_{m-k}(A)$. 
Lemma 3.1. We have $(f \mid A)_{*}[A]=0$ in $\check{H}_{m-k}(B)$.

Proof. Set $M_{2}(f)=\left\{x \in M \mid f^{-1}(f(x))=\{x, y\}\right.$ with $x \neq y$ and $d f_{x}, d f_{y}$ are injective $\}$. Note that $M_{2}(f)$ is nothing but the open dense subset $U$ of $A$ described above $([\mathrm{R}])$. Set $A^{\prime}=A-M_{2}(f)$. Then we see that $A^{\prime}=\Sigma(f) \cup M_{3}(f)$, where $M_{3}(f)=\left\{x \in M \mid f^{-1}(f(x))\right.$ contains at least 3 elements $\}$. Setting $S(f)=$ $f^{-1}(f(\Sigma(f)))$, we see that $f\left(A^{\prime}\right)=f(\Sigma(f)) \cup f\left(M_{3}(f)-S(f)\right)$. Since $j^{1} f$ is transverse to $\Sigma^{i}$, we see that $\Sigma(f)$ is a finite disjoint union of differentiable submanifolds of dimensions at most $m-k-1$. Furthermore, since $f \mid(M-S(f))$ is a proper immersion with normal crossings, $M_{3}(f)-S(f)=M_{3}(f \mid(M-S(f)))$ is a disjoint union of countable number of manifolds of dimensions at most $m-2 k \leq m-k-1$. Thus, using [HW, Theorem III 2], we see that the topological dimension of $f\left(A^{\prime}\right)$ is at most $m-k-1$. This implies that $\check{H}_{m-k}\left(f\left(A^{\prime}\right)\right)=0$.

Now consider the following commutative diagram with exact rows:

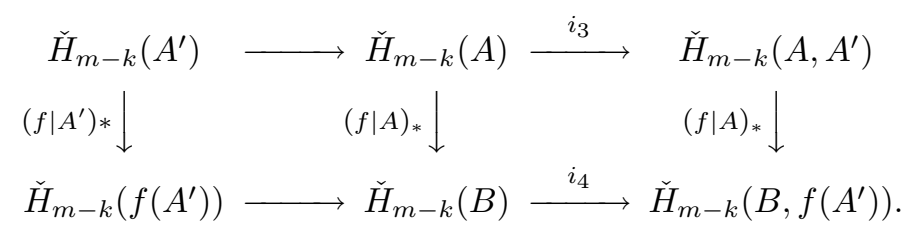

Since $i_{4}$ is injective, in order to show that $(f \mid A)_{*}[A]=0$ in $\check{H}_{m-k}(B)$, we have only to show that $(f \mid A)_{*} \circ i_{3}([A])=0$ in $\check{H}_{m-k}\left(B, f\left(A^{\prime}\right)\right)$.

Lemma 3.2. Let $(X, Y)$ be a relative manifold; i.e., $X$ is compact and Hausdorff, $Y$ is closed in $X$ and $X-Y$ is a (topological) manifold. Then we have a canonical isomorphism $\check{H}_{i}(X, Y) \cong H_{i}^{c}(X-Y)$.

Proof. Since $X-Y$ is a manifold, there exists a sequence of compact codimension-0 submanifolds $K_{0} \subset K_{1} \subset K_{2} \subset \cdots$ such that $K_{j} \subset \operatorname{Int} K_{j+1}$ and that $\cup_{j} K_{j}=$ $X-Y$. Then we have the isomorphism $\check{H}_{i}(X, Y) \cong \lim _{\longleftarrow} \check{H}_{i}\left(X, X-\operatorname{Int} K_{j}\right)$ by the continuity of the Čech homology theory (see [ES, p. 261], for example). The right hand side is isomorphic to $\lim _{\longleftarrow} \breve{H}_{i}\left(X, X-K_{j}\right)$, which is nothing but $\breve{H}_{i}^{c}(X-Y)$ by the definition, where $\check{H}_{*}^{c}$ denotes the Čech homology with compact carriers. Since $X-Y$ is a manifold, which is an ANR, we have the canonical isomorphism $\check{H}_{i}^{c}(X-Y) \cong H_{i}^{c}(X-Y)$. This completes the proof.

Now since $\left(A, A^{\prime}\right)$ and $\left(B, f\left(A^{\prime}\right)\right)$ are relative manifolds by $[\mathrm{R}]$, we have the following commutative diagram, where the horizontal maps are isomorphisms by 
Lemma 3.2:

$$
\begin{array}{ccc}
\check{H}_{m-k}\left(A, A^{\prime}\right) & \stackrel{\theta_{1}}{\longrightarrow} & H_{m-k}^{c}\left(A-A^{\prime}\right) \\
(f \mid A)_{*} \downarrow & & \downarrow\left(f \mid\left(A-A^{\prime}\right)\right)_{*} \\
\check{H}_{m-k}\left(B, f\left(A^{\prime}\right)\right) \stackrel{\theta_{2}}{\longrightarrow} & H_{m-k}^{c}\left(B-f\left(A^{\prime}\right)\right) .
\end{array}
$$

Since $f \mid\left(A-A^{\prime}\right)$ is a double covering, we see easily that $\left(f \mid\left(A-A^{\prime}\right)\right)_{*} \circ \theta_{1} \circ i_{3}([A])=0$ in $H_{m-k}^{c}\left(B-f\left(A^{\prime}\right)\right)$. Thus we have $(f \mid A)_{*} \circ i_{3}([A])=0$. This completes the proof of Lemma 3.1.

Remark 3.3. The following alternative proof of Lemma 3.1 has been communicated to the authors by the referee. Let $F_{2}(M)$ be the "fat square" of $M$; i.e., it is the manifold obtained by blowing up the diagonal in $M \times M$ (see [R]). Furthermore, let $\pi: F_{2}(M) \rightarrow M$ be the natural map and $\tau: F_{2}(M) \rightarrow F_{2}(M)$ the natural involution induced by the map which permutes the factors of $M \times M$. Then, as is shown in [R], there exist a closed $(m-k)$-dimensional submanifold $\tilde{M}_{2}(f)$ of $F_{2}(M)$ and an open dense subset $\tilde{M}_{2}^{\circ}(f)$ of $\tilde{M}_{2}(f)$ such that $\pi\left(\tilde{M}_{2}(f)\right)=A=\overline{M(f)}$ and that $\pi \mid \tilde{M}_{2}^{\circ}(f)$ is an embedding whose image is dense in $A$. In other words, $\tilde{M}_{2}(f)$ is a desingularization of $A$. Recall that the fundamental class $[A] \in H_{m-k}(A)$ is nothing but $\pi_{*}\left[\tilde{M}_{2}(f)\right]$, where $\left[\tilde{M}_{2}(f)\right] \in H_{m-k}\left(\tilde{M}_{2}(f)\right)$ is the usual fundamental class of the manifold $\tilde{M}_{2}(f)$. Furthermore, it is easily seen from the construction that $\tilde{M}_{2}(f)$ is invariant under $\tau$. Moreover, the quotient space $\tilde{B}=\tilde{M}_{2}(f) / \tau$ is a manifold possibly with boundary and the quotient map $\gamma: \tilde{M}_{2}(f) \rightarrow \tilde{B}$ is a double branched covering branched along the boundary of $\tilde{B}$. Now consider the following commutative diagram:

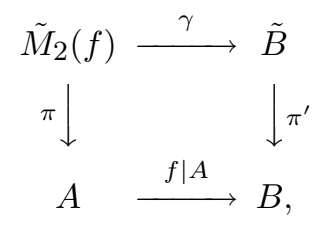

where $\pi^{\prime}: \tilde{B} \rightarrow B$ is the natural map induced by $\pi \mid \tilde{M}_{2}(f)$. Since $\gamma_{*}\left[\tilde{M}_{2}(f)\right]=0$ in $H_{m-k}(\tilde{B})$, we see that $(f \mid A)_{*}[A]=\pi_{*}^{\prime}\left(\gamma_{*}\left[\tilde{M}_{2}(f)\right]\right)=0$. Then it follows easily that $(f \mid A)_{*}[A]=0$ also in $\tilde{H}_{m-k}(B)$. This completes the alternative proof.

Now we consider $j_{*}[A] \in \check{H}_{m-k}(M)$. Since $M$ is a manifold, which is an ANR, we have the canonical isomorphism $\check{H}_{m-k}(M) \cong H_{m-k}(M)$. Then, by the commutative diagram

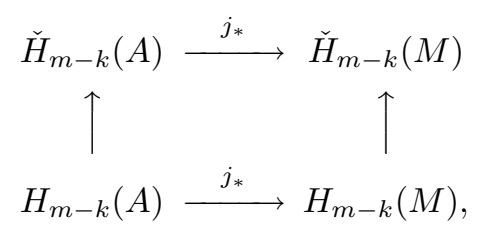


where the vertical homomorphisms are the natural ones, we see that $j_{*}[A] \in$ $\check{H}_{m-k}(M)$ with $[A] \in \check{H}_{m-k}(A)$ is identified naturally with $j_{*}[A] \in H_{m-k}(M)$ with $[A] \in H_{m-k}(A)$. Then by $[\mathrm{R}]$, we see that $j_{*}[A]=D_{M}\left(w_{k}(f)-v(f)\right)$, where $D_{M}: H^{k}(M) \rightarrow H_{m-k}(M)$ is the Poincaré duality isomorphism.

Now suppose that $w_{k}(f)=v(f)$. Then we see that $[A]$ is a non-zero element in $\operatorname{ker} \alpha$, which implies that

$$
\operatorname{dim} \check{H}_{m-k+1}(M)<\operatorname{dim} \check{H}_{m-k+1}(f(M)) .
$$

Hence we have proved that if $f$ is not a differentiable embedding, then either $w_{k}(f) \neq v(f)$ or $\check{\beta}_{m-k+1}(M) \neq \check{\beta}_{m-k+1}(f(M))$.

If $f$ is a differentiable embedding, then we know that $w_{k}(f)=v(f)$ and we trivially have $\breve{\beta}_{m-k+1}(M)=\breve{\beta}_{m-k+1}(f(M))$. This completes the proof of Theorem 2.2 .

Remark 3.4. We do not know if Theorem 2.2 holds even if we replace $\check{\beta}_{m-k+1}$ by the usual $(m-k+1)$-th Betti number with respect to the singular homology. This is true, if $f(M)$ is an ANR. For example, if $f$ is generic in the sense of [GWPL], then $f(M)$ is triangulable and is an ANR. For details, see [BS2].

Furthermore, we do not know if Theorem 2.2 holds when $M$ is noncompact and $f$ is proper. Note that in the proof above, we have essentially used the compactness of $M$ in order to guarantee that the Cech homology sequences for $(M, A)$ and $(f(M), B)$ are exact. Note also that the corresponding result for generic maps in the sense of [GWPL] does hold (see [BS2, §4]).

Remark 3.5. In [N, §3], Nuño Ballesteros considers a class of $C^{\infty}$ proper maps for which the topological dimension of $f(A)$ is smaller than or equal to $m-1$, where he considers the case $k=1$. Although his class is residual in $C_{\mathrm{pr}}^{\infty}(M, N)$, maps of this class should satisfy strong transversality conditions, and our class of the maps which are generic for the double points is much richer. See the example in $\S 4$, for example.

Proof of Corollary 2.4. First note that, since $H_{k}(N)=0$, the hypotheses of Corollary 2.3 are satisfied for $f$. Now consider the following exact sequence of singular cohomology:

$$
\tilde{H}^{2 k-2}(N) \rightarrow \tilde{H}^{2 k-2}(N-f(M)) \rightarrow H^{2 k-1}(N, N-f(M)) \rightarrow \tilde{H}^{2 k-1}(N) .
$$

Note that $\tilde{H}^{2 k-2}(N)=0$ and $\tilde{H}^{2 k-1}(N)=0$ by our assumptions and the universal coefficient theorem. Furthermore, we have a canonical isomorphism $H^{2 k-1}(N, N-$ $f(M)) \cong \check{H}_{m-k+1}(f(M))$ (see [Gr, p. 179]). Thus we have $\tilde{\beta}_{2 k-2}(N-f(M))=$ $\check{\beta}_{m-k+1}(f(M))$. Note also that $\check{\beta}_{m-k+1}(M)=\beta_{m-k+1}(M)=\beta_{k-1}(M)$ by Poincaré duality. Then, combining this with Corollary 2.3, we obtain the conclusion. This completes the proof of Corollary 2.4. 
In the codimension-1 case, using a result of [Sae1], we obtain the following, which is slightly stronger than Corollary 2.6.

Theorem 3.6. Let $f: M \rightarrow N$ be a proper map of class $C^{2}$ which is generic for the double points, where $M$ and $N$ are connected orientable manifolds of dimensions $m$ and $m+1$ respectively. We suppose that $H_{1}(N ; \mathbf{Z})$ is a torsion group (i.e., every element has finite order) or that $f_{*}[M]$ is a torsion element in $H_{m}^{c}(N ; \mathbf{Z})$, where $[M] \in H_{m}^{c}(M ; \mathbf{Z})$ is a fundamental class of $M$. Then $f$ is a differentiable embedding if and only if $\beta_{0}(N-f(M))=2$.

Proof. First suppose that $f$ is not a differentiable embedding. Then we have $M(f) \cup \Sigma(f) \neq \emptyset$. On the other hand, by [R], we have $M(f) \cup \Sigma(f)=\overline{M_{2}(f)}$. Thus $M_{2}(f) \neq \emptyset$, which implies that $f$ has a normal crossing point of multiplicity 2 . Then by [Sae1] together with our hypotheses, we have $\beta_{0}(N-f(M)) \geq 2+1=3$.

If $f$ is a differentiable embedding, we see easily that $\beta_{0}(N-f(M)) \leq 2$ and we also have $\beta_{0}(N-f(M)) \geq 2$ by [Sae1]. This completes the proof.

Remark 3.7. In [Hi], Hirsch has shown that, if $f: M \rightarrow N$ is a codimension$k$ proper $C^{2}$-immersion and $H_{k}(N)=0$, then $H_{k-1}(N-f(M))$ is non-trivial. This is valid also for maps of class $C^{2}$ which are generic for the double points. More precisely, let $f: M \rightarrow N$ be a map of class $C^{2}$ which is generic for the double points, where $M$ is a closed $m$-dimensional manifold, $N$ is an $n$-dimensional manifold with $k=n-m>0, \operatorname{dim} H_{k-1}(N)$ is finite, and $H_{k}(N)=0$. Then we have the following.

(1) We always have

$$
\begin{aligned}
\beta_{k-1}(N-f(M)) & \left.=\beta_{k-1}(N)+\check{\beta}_{m}(f(M))\right) \\
& \geq \beta_{k-1}(N)+\beta_{0}(M) .
\end{aligned}
$$

(2) When $k=1$ and $M$ is orientable, the equality holds in (1) if and only if $f$ is a differentiable embedding.

(3) When $k \geq 2$, the equality in (1) always holds.

We can prove the above facts using the techniques developed in this section and we omit the proof.

\section{Example}

In this section we give an example of a map which is generic for the double points and whose image is not an ANR. In particular, this is an example of a map which is generic for the double points but not generic in the sense of [GWPL, BS2].

We will construct a smooth map $f:\left(S^{2} \times S^{1}\right) \sharp\left(S^{2} \times S^{1}\right) \rightarrow S^{3} \times S^{1}$ with the desired property. Let $\varphi: S^{2} \rightarrow S^{3}$ be the stable map whose image is as 


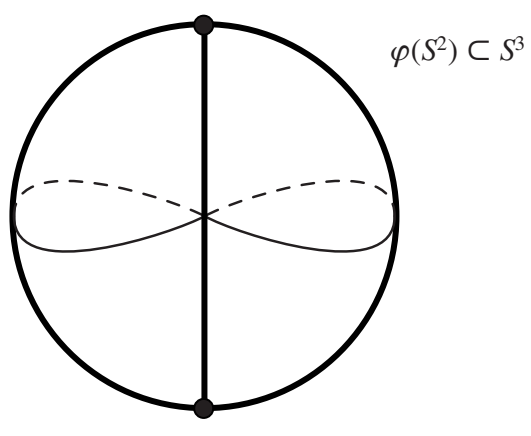

Figure 1
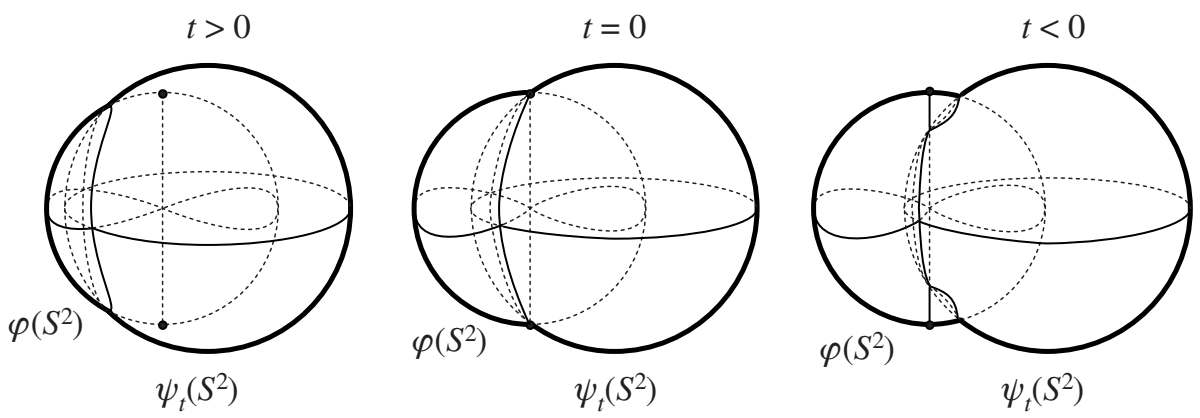

Figure 2

in Figure 1. Note that $\varphi$ has exactly two singular points, which are cross cap points. Now consider a smooth family of embeddings $\psi_{t}: S^{2} \rightarrow S^{3}(t \in \mathbf{R})$ whose images intersect $\varphi\left(S^{2}\right)$ as in Figure 2. Note that, for every $t$, the map $\varphi \cup \psi_{t}: S^{2} \cup S^{2} \rightarrow S^{3}$ is generic for the double points. Now choose an arbitrary smooth function $\alpha: S^{1} \rightarrow \mathbf{R}$ and consider the smooth map

$$
F=(\varphi \times \text { id }) \cup \Psi:\left(S^{2} \times S^{1}\right) \cup\left(S^{2} \times S^{1}\right) \longrightarrow S^{3} \times S^{1},
$$

where $\Psi: S^{2} \times S^{1} \rightarrow S^{3} \times S^{1}$ is the smooth map defined by $\Psi(x, y)=\left(\psi_{\alpha(y)}(x), y\right)$. Finally we perform the connected sum operation to $F$ so that it creates no new multiple points nor singular points. The resulting map is denoted by $f:\left(S^{2} \times\right.$ $\left.S^{1}\right) \sharp\left(S^{2} \times S^{1}\right) \rightarrow S^{3} \times S^{1}$. Note that $f$ is always generic for the double points. However, $f$ is not generic in the sense of [GWPL] if $\alpha\left(S^{1}\right)$ contains 0. Furthermore, the closure $A=\overline{M(f)}$ of the self-intersection set of $f$ consists of "two parts", each of which is a 1-parameter family of 1-dimensional objects parametrized by $S^{1}$. More precisely $A=\cup_{y \in S^{1}}\left(A_{y} \cup B_{y}\right)$, where $A_{y}$ and $B_{y}$ are as in Figure 3 . Note that $\sigma_{i} \in \Sigma(f)(i=1,2, \ldots, 6), f\left(s_{1}\right)=f\left(\sigma_{3}\right), f\left(s_{2}\right)=f\left(\sigma_{4}\right), f\left(t_{1}\right)=f\left(t_{3}\right)=$ $f\left(t_{5}\right)$, and $f\left(t_{2}\right)=f\left(t_{4}\right)=f\left(t_{6}\right)$. For example, if we take a smooth function 


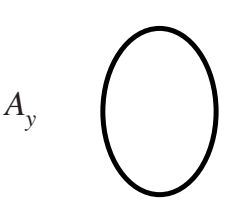

$$
\alpha(y)>0
$$

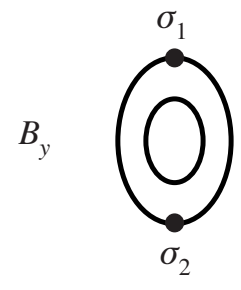

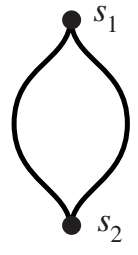

$\alpha(y)=0$

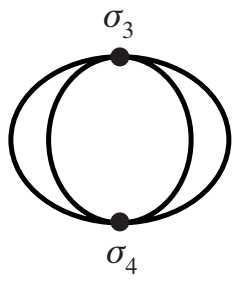

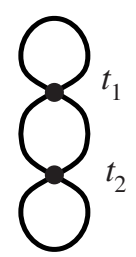

$\alpha(y)<0$

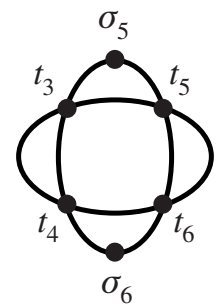

Figure 3

$\alpha: S^{1} \rightarrow \mathbf{R}$ such that $\alpha^{-1}((-\infty, 0))$ has infinitely many components, then $H_{*}(A)$ is not finitely generated, and consequently $A$ is not an ANR. In this case, the image $f\left(\left(S^{2} \times S^{1}\right) \sharp\left(S^{2} \times S^{1}\right)\right)$ is not an ANR, either. If we take a smooth function $\alpha: S^{1} \rightarrow \mathbf{R}$ such that $\alpha(y) \geq 0$ for all $y \in S^{1}$ and $\alpha^{-1}(0)$ is a Cantor set, we see that $A-M_{2}(f)$ has uncountably many connected components.

More generally, using a smooth function $\beta: M \rightarrow \mathbf{R}$ of a smooth closed manifold $M$, we can construct a smooth map $\left(S^{2} \times M\right) \sharp\left(S^{2} \times M\right) \rightarrow S^{3} \times M$ which is generic for the double points but which is not generic in the sense of [GWPL].

\section{References}

[BMS1] C. Biasi, W. Motta and O. Saeki, A note on separation properties of codimension-1 immersions with normal crossings, Topology Appl. 52 (1993), 81-87.

[BMS2] C. Biasi, W. Motta and O. Saeki, A remark on the separation by immersions in codimension-1, Topology Appl. 61 (1995), 179-186.

[BR] C. Biasi and M. C. Romero Fuster, A converse of the Jordan-Brouwer theorem, Illinois J. Math. 36 (1992), 500-504.

[BS1] C. Biasi and O. Saeki, On the Betti number of the image of a codimension- $k$ immersion with normal crossings, Proc. Amer. Math. Soc. 123 (1995), 3549-3554.

[BS2] C. Biasi and O. Saeki, On the self-intersection set and the image of a generic map, to appear in Math. Scand.

[BS3] C. Biasi and O. Saeki, On bordism invariance of an obstruction to topological embeddings, Osaka J. Math. 33 (1996), 729-735. 
[C] P. T. Church, On points of Jacobian rank k. II, Proc. Amer. Math. Soc. 16 (1965), 1035-1038.

[D] E. Dyer, Cohomology theories, W. A. Benjamin, Inc., New York, Amsterdam 1969.

[ES] S. Eilenberg and N. Steenrod, Foundations of algebraic topology, Princeton Univ. Press, Princeton, New Jersey 1952.

[GWPL] C. G. Gibson, K. Wirthmüller, A. A. du Plessis and E. J. N. Looijenga, Topological stability of smooth mappings, Lecture Notes in Math. 552, Springer-Verlag, Berlin, Heidelberg, New York 1976.

[Gr] M. J. Greenberg, Lectures on algebraic topology, Mathematics Lecture Notes, W. A. Benjamin, Inc., New York, Amsterdam 1967.

[He] R. Herbert, Multiple points of immersed manifolds, Memoirs Amer. Math. Soc. 34 (250), 1981.

[Hi] M. D. Hirsch, The complement of a codimension- $k$ immersion, Proc. Camb. Phil. Soc. 107 (1990), 103-107.

[HW] W. Hurewicz and H. Wallman, Dimension theory, Princeton Math. Series 4, Princeton Univ. Press, Princeton 1948.

[K] G. M. Kelly, The exactness of Čech homology over a vector space, Proc. Camb. Phil. Soc. 57 (1961), 428-429.

[LS] R. Lashof and S. Smale, Self-intersections of immersed manifolds, J. Math. and Mech. 8 (1959), 143-157.

[MS] J. Milnor and J. Stasheff, Characteristic classes, Ann. of Math. Stud. 76, Princeton Univ. Press, Princeton 1974.

[N] J. J. Nuño Ballesteros, Counting the connected components of the complement of the image of a codimension 1 map, Compositio Math. 93 (1994), 37-47.

[R] F. Ronga, 'La classe duale aux points doubles' d'une application, Compositio Math. 27 (1973), 223-232.

[Sae1] O. Saeki, Separation by a codimension-1 map with a normal crossing point, Geom. Dedicata 57 (1995), 235-247.

[Sae2] O. Saeki, A converse of the Jordan-Brouwer Theorem for quasi-regular immersions, Illinois J. Math. 40 (1996), 108-114.

[Sar] A. Sard, Hausdorff measure of critical images on Banach manifolds, Amer. J. Math. 87 (1965), 158-174.

[Sp] E. H. Spanier, Algebraic topology, TATA McGraw-Hill Publishing Company Ltd., Bombay, New Delhi 1966.

[Wa] A. H. Wallace, Algebraic topology: homology and cohomology, W. A. Benjamin, Inc., New York 1970.

[Wh] H. Whitney, On the topology of differentiable manifolds, Lectures on Topology, Univ. Michigan Press 1941.

Carlos Biasi

Departamento de Matemática

ICMSC-USP

Caixa Postal 668

13560-970 São Carlos, SP

Brazil

E-mail: biasi@ICMSC.SC.USP.BR
Osamu Saeki

Department of Mathematics

Faculty of Science

Hiroshima University

Higashi-Hiroshima 739

Japan

E-mail: saeki@top2.math.sci.hiroshima-u.ac.jp

(Received: January 3, 1996) 